\title{
Constrained Detection for Noncoherent Nonlinear Multiuser Communications
}

\author{
Rajnish Sinha \\ WINLAB, Rutgers University \\ 73 Brett Road \\ Piscataway NJ 08854 \\ sinhar@winlab.rutgers.edu
}

\author{
Aylin Yener \\ EECS, Lehigh University \\ 19 Memorial Drive West \\ Bethlehem, PA 18015-3084 \\ yener@eecs.lehigh.edu
}

\author{
Roy D. Yates \\ WINLAB, Rutgers University \\ 73 Brett Road \\ Piscataway NJ 08854 \\ ryates@winlab.rutgers.edu
}

\begin{abstract}
We consider noncoherent multiuser detection techniques for a system with users employing nonlinear modulation using non-orthogonal signals. Our aim is to investigate nearoptimum noncoherent multiuser detection techniques that utilize the structure of the received signals while retaining reasonable complexity. We explore detectors that are derived using nonlinear programming approximations of the maximum likelihood multiuser detector as well as soft interference cancellers. We also propose a class of detectors called the partial detectors which improve the performance of the decorrelator and MMSE type detectors. We evaluate the proposed detectors' performance to provide numerical comparisons.
\end{abstract}

\section{Introduction}

Noncoherent detection is often necessary for systems for which the phase estimation is difficult due to rapid changes in the channel conditions [5]. Nonlinear $M$-ary modulation in a multiuser setting and coherent and noncoherent multiuser detection techniques for such systems have also become popular recently, using orthogonal or non-orthogonal (correlated) pulses to transmit different messages of the different users $[7,9]$.

The non-orthogonal multipulse modulation for a multiuser system is investigated in [7] where each user chooses one of several waveforms to send a message. When correlated waveforms are used to transmit the messages of the users, multiuser interference issues arise since the receiver observes the superposition of all users' transmissions. Similar to its coherent, linear modulation counterpart [8], the maximum likelihood (ML) detector that estimates all users' messages jointly for noncoherent communications with nonlinear modulation, has high complexity. References [7, 2] propose several less complex noncoherent multiuser receivers that consist of a decorrelating or a mini- mum mean squared error (MMSE) pre-filter for interference suppression followed by a non-coherent decision device that estimates the message sent by each user. These detectors will henceforth be referred to as the full detectors to distinguish them from the partial detectors proposed in this paper.

The transmitted signals in the non-orthogonal multipulse modulation scheme have a special structure, and in this paper, we investigate the effect of incorporating this special structure into the detection algorithms for noncoherent nonlinear multiuser communications. Our efforts can be broadly classified in three categories: constrained optimization, soft interference cancellation, and partial detection.

The constrained detectors use nonlinear programming approximations of the ML multiuser detector (pre-filter) [10]. Several SIC (Soft Interference Cancellation) detectors are also investigated, some of which yield better performance than the full detectors in the near-far scenario. The partial detectors proposed in this paper also use the structure of the signal and yield improved performance over the full detectors. We evaluate the performance of all the proposed detectors and compare the results obtained. We also compare our results with those of [2].

\section{System Model}

We consider a synchronous CDMA system with processing gain $N, K$ active users, and a signaling scheme where each user transmits one of $M$ signals. A discrete-time model can be obtained by projecting the received signal onto an $N$-dimensional orthonormal basis. Using the pseudo-linear representation introduced in [7], we view the signal space as being an expanded signal space spanned by the $M K$ signals, $M$ messages for each of the $K$ users. We concentrate on cases where the possible waveforms for all messages of all the users are linearly independent. The channel is assumed to be AWGN, and the receiver observes a superposition of the $K$ signals.

Let $m_{k}$ be the desired message of the $k$ th user. We define 
the vector $\mathbf{b}_{k}=\left[b_{k, 1} \cdots b_{k, M}\right]^{\top} \in F$, where

$$
b_{k, m}= \begin{cases}1 & m=m_{k} \\ 0 & \text { otherwise }\end{cases}
$$

and $F=\left\{\left[\begin{array}{llll}1 & 0 & \cdots & 0\end{array}\right]^{\top}, \ldots,\left[\begin{array}{llll}0 & \cdots & 0 & 1\end{array}\right]^{\top}\right\}$. The $N \times 1$ vector $s_{k m}$ represents the $k$ th user's signature corresponding to message $m$. The $N \times M$ signature matrix of the $k$ th user is denoted as $\mathbf{S}_{k} \triangleq\left[\mathbf{s}_{k 1} \cdots \mathbf{s}_{k M}\right] ; A_{k m}$ and $\phi_{k m}$ represent the amplitude and phase of user $k$ associated with message $m ; \mathbf{A}_{k} \triangleq \operatorname{diag}\left[A_{k 1}, \ldots, A_{k M}\right]$ and $\boldsymbol{\Phi}_{k} \triangleq \operatorname{diag}\left[e^{j \phi_{k 1}}, \ldots, e^{j \phi_{k M}}\right]$ are $M \times M$ diagonal matrices representing the amplitudes and phases of all the $M$ messages of user $k$. The phases are assumed to be independent and uniformly distributed over $[0,2 \pi]$. The received vector at the output of the bank of matched filters can be written as

$$
\mathbf{r}=\sum_{k=1}^{K} \mathbf{S}_{k} \mathbf{A}_{k} \mathbf{\Phi}_{k} \mathbf{b}_{k}+\mathbf{n}
$$

where $\mathbf{n}$ is the AWGN vector. Further, $\mathbf{r}$ can be expressed in terms of the $M K \times 1$ vector $\mathbf{b}=\left[\mathbf{b}_{1}^{\top} \cdots \mathbf{b}_{K}^{\top}\right]^{\top}$, the $N \times M K$ matrix $\mathbf{S} \triangleq\left[\mathbf{S}_{1} \ldots \mathbf{S}_{K}\right]$, the $M K \times M K$ matrices $\mathbf{A} \triangleq \operatorname{diag}\left[\mathbf{A}_{1}, \ldots, \mathbf{A}_{K}\right]$ and $\boldsymbol{\Phi} \triangleq \operatorname{diag}\left[\boldsymbol{\Phi}_{1}, \ldots, \mathbf{\Phi}_{K}\right]$ as

$$
\mathbf{r}=\mathbf{S A} \boldsymbol{\Phi} \mathbf{b}+\mathbf{n}
$$

The vector $\mathbf{b} \in \mathcal{F}$ where $\mathcal{F}=F \times F \times \ldots \times F$. The aim of the multiuser detector is to recover the message vector $b$. The ML estimate of $b$ given $r$, and known $A$ and $\Phi$, is the solution to the optimum multiuser detector [8]. The estimate may be written as

$$
\hat{\mathbf{b}}=\arg \min _{\mathbf{b} \in \mathcal{F}}\|\mathbf{r}-\mathbf{S A} \mathbf{\Phi} \mathbf{b}\|^{2}
$$

Note that (3) describes the coherent detector since it assumes the knowledge of $\mathbf{\Phi}$.

\section{Noncoherent Multiuser Detection}

Consider the case where the amplitudes, $\mathbf{A}$, are known at the receiver as in (3), but both $\Phi$ and $b$ are unknown. In this case, we can estimate them jointly as $\mathbf{x}=\boldsymbol{\Phi} \mathbf{b}$, and the corresponding detector will be referred to as the Jointly $O p$ timum Detector. If we define the set $G$ as

$$
G=\left\{\left[\begin{array}{lllll}
e^{j \phi_{1}} & 0 & \cdots & 0
\end{array}\right]^{\top}, \ldots,\left[\begin{array}{llll}
0 & \cdots & 0 & e^{j \phi_{M}}
\end{array}\right]^{\top}\right\}
$$

then, $\mathrm{x} \in \mathcal{G}$, where $\mathcal{G}=G \times G \times \ldots \times G$. The jointly optimal estimate $\hat{\mathbf{x}}$ is the solution to

$$
\begin{array}{ll}
\operatorname{minimize} & \|\mathbf{r}-\mathbf{S A x}\|^{2} \\
\text { subject to } & \mathbf{x} \in \mathcal{G}
\end{array}
$$

The implementation of this detector requires an exhaustive search. For a given $\mathrm{b}$, let the vector $\phi=\left[e^{j \phi_{1}} \cdots e^{j \phi_{K}}\right]^{T}$ represent the phases corresponding to the $K$ non-zero entries of $\mathbf{b}$. Then, in terms of $f(\mathbf{r} \mid \mathbf{b}, \phi)$ the conditional PDF of $\mathbf{r}$, the joint ML estimate of $\hat{b}$ and $\hat{\phi}$ is

$$
(\hat{\mathbf{b}}, \hat{\boldsymbol{\phi}})=\arg \max _{\mathbf{b}} \max _{\phi} f(\mathbf{r} \mid \mathbf{b}, \boldsymbol{\phi})
$$

Since each of the elements of $\phi$ lie on a unit circle, the inner maximization in (6) above is over a non-convex set and hence there is no guarantee of finding the global minimum. However, if we relax the constraints and allow each of the elements of $\phi$ to lie within the unit circle, then the set is convex. This detector will be referred to as the Joint Detector.

Next consider the decorrelative and the MMSE detectors. Let

$$
\mathbf{y}=\mathbf{S}^{H} \mathbf{r}=\mathbf{R A} \mathbf{x}+\mathbf{S}^{H} \mathbf{n}
$$

The decorrelative detector consists of two stages. The first stage implements the decorrelative pre-filter [7] as follows

$$
\hat{\mathbf{x}}=(\mathbf{R A})^{-1} \mathbf{y}=\mathbf{x}+(\mathbf{R A})^{-1} \mathbf{S}^{H} \mathbf{n}
$$

In the second stage, to obtain an estimate $\hat{m}_{k}$ of the $k$ th user's message, the Maximum Magnitude (MM) rule is applied as suggested in $[2,3]$, where

$$
\hat{m}_{k}=\arg \max _{m \in\{1, \ldots, M\}}\left|\hat{x}_{k m}\right|^{2}
$$

The MMSE pre-filter on the other hand, applies the matrix transformation $\mathbf{C}^{H}$ to the output of the matched filters $\mathbf{r}$ [2]. It minimizes the mean-squared error $E\left[\left\|\mathbf{C}^{H} \mathbf{r}-\mathbf{x}\right\|^{2}\right]$, and the solution is

$$
\mathbf{C}=\mathbf{H}^{-1} \mathbf{S A E}
$$

where $\mathbf{H}=E\left[\mathbf{r r}^{H}\right]=\mathbf{S A E A S} \mathbf{S}^{H}+\sigma^{2} \mathbf{I}_{N}$ and $\mathbf{E}=$ $E\left[\mathbf{x} \mathbf{x}^{H}\right]=(1 / M) \mathbf{I}_{M K}$, where $\mathbf{I}_{n}$ is the identity matrix of dimension $n$. The estimate of the desired vector $\mathrm{x}$ is obtained as $\hat{\mathbf{x}}=\mathbf{C}^{H} \mathbf{r}$, and is sub-optimally decoupled in order to yield statistics $\hat{\mathbf{x}}_{k}$ for the $k$ th user, where $\hat{\mathbf{x}}_{k}=\mathbf{C}_{k}^{H} \mathbf{r}$ and $\mathbf{C}_{k}=\mathbf{H}^{-1} \mathbf{S}_{k} \mathbf{A}_{k} \mathbf{E}_{k}$. The estimate of the $k$ th user's message is then obtained by applying the MM rule to the vector $\mathbf{x}_{k}$.

\section{Constrained Noncoherent Multiuser Detec- tion}

Due to the high complexity associated with the ML detector, approximations are obtained by solving an easier problem. The easier problem is a relaxation of the original problem and can be solved efficiently. In this section, an effort is made to incorporate the structure of the signals into the relaxation to try and improve performance. 
Since $\mathbf{x}_{k} \in G, \mathbf{x}_{k}^{H} \mathbf{x}_{k}=1$ for all $k$. We will call this constraint a local constraint and explore its relaxation. Also, since $\mathbf{x} \in \mathcal{G}, \mathbf{x}^{H} \mathbf{x}=K$. This constraint will be termed a global constraint, and its relaxation will also be explored. Note that the constraints are not representative of the complete structure of the signal since there are other vectors that satisfy the constraints but are not in the feasible set of transmitted signals. For example, $\mathbf{x}_{k}=[1 / \sqrt{M}, \ldots, 1 / \sqrt{M}]^{T}$ satisfies $\mathbf{x}_{k}^{H} \mathbf{x}_{k}=1$, but does not belong to $G$.

- Local Constraint

This is the first constrained optimization problem we investigate as an approximation to the original ML problem. Since the objective function $\|\mathbf{r}-\mathbf{S A x}\|^{2}$ is convex in $\mathbf{x}$, it has a global minimum. However, the constraint $\mathbf{x}_{k}^{H} \mathbf{x}_{k}=1$ is not a convex set and hence there is no guarantee that the global minimum will be found. If we relax this constraint to be $\mathbf{x}_{k}^{H} \mathbf{x}_{k} \leq 1$, which represents the interior of a unit sphere, we are guaranteed to find the global minimum. We can then use nonlinear programming techniques [1] to find this optimum value. The estimate $\hat{\mathbf{x}}$ is the solution to

$$
\begin{array}{ll}
\operatorname{minimize} & \|\mathbf{r}-\mathbf{S A} \mathbf{x}\|^{2} \\
\text { subject to } & \left\|\mathbf{x}_{k}\right\|^{2} \leq 1 \quad k=1, \ldots, K
\end{array}
$$

followed by the MM rule to obtain $\hat{m}_{k}$.

\section{- Global Constraint}

The constraint $\mathbf{x}^{H} \mathbf{x}=K$ is also non-convex thus if we relax it to be the convex set $x^{H} \mathbf{x} \leq K$, we are guaranteed to find the global optimum. The estimate $\hat{\mathbf{x}}$ is the solution to the following optimization problem

$$
\begin{array}{ll}
\operatorname{minimize} & \|\mathbf{r}-\mathbf{S A x}\|^{2} \\
\text { subject to } & \|\mathbf{x}\|^{2} \leq K
\end{array}
$$

The convex set $\|\mathbf{x}\|^{2} \leq K$ may be thought of as the interior of a sphere of radius $\sqrt{K}$. The MM rule is applied to $\hat{\mathbf{x}}_{k}$ to obtain $\hat{m}_{k}$ as in (9) above. It can also be shown that the solution to the optimization problem in (12) results in exactly the same Generalized MMSE detector as derived in [11, 10]. Hence, the relaxation in (12) can be termed as the generalized MMSE solution.

\section{Soft Interference Cancellation (SIC)}

In contrast to the optimum multiuser detector, suboptimal multistage detectors have a relatively lower complexity [6]. This section explores different noncoherent realizations of the decision directed, nonlinear detectors proposed in $[4,6]$. All the implementations here use the decorrelator output in the first stage to obtain sufficient statistics, followed by multiple stages of processing of these sufficient statistics. The goal once again is to obtain $\hat{\mathbf{x}}$. To obtain an estimate for the current user's message, soft estimates are used to reconstruct the interference and are then subtracted off from the desired user's matched filter output. Since the high energy users are successfully cancelled in the subsequent stages, these detectors perform very well in near-far scenarios.

\section{- Serial SIC}

In this detector, each element of $\mathbf{x}$ is found iteratively using the Gauss-Seidel iteration [1]. In the first step, at the $[n+1]$ st stage, the $k$ th user's $i$ th element is determined as follows

$$
\begin{aligned}
& x_{i}[n+1]= \\
& \frac{1}{A_{i}}\left(y_{i}-\sum_{j=1}^{i-1} R_{i j} A_{j} x_{j}[n+1]-\sum_{j=i+1}^{M K} R_{i j} A_{j} x_{j}[n]\right)
\end{aligned}
$$

All the $M$ entries of user $k$ are thus iteratively determined, and then, in the second step, the entry with the maximum magnitude is selected as

$x_{i}[n+1]= \begin{cases}x_{i}[n+1] & \text { if }\left|x_{i}[n+1]\right| \geq\left|x_{j}[n+1]\right| \\ 0 & \text { otherwise }\end{cases}$

This vector estimate is then used by the $(k+1)$ st user in (13) above for estimating its vector, and so on.

\section{- Clipped SIC}

The first step is exactly the same as in (13) above. In the second step, we incorporate the relaxed constraint $\left|x_{i}\right| \leq 1$ by clipping in accordance with the following rule:

$$
x_{i}[n+1]= \begin{cases}\left.x_{i}[n+1]\right] & \text { if }\left|x_{i}[n+1]\right| \leq 1 \\ \frac{\left.x_{i} \mid n+1\right]}{\left|x_{i}[n+1]\right|} & \text { otherwise }\end{cases}
$$

\section{- Parallel SIC}

Here, instead of estimating each element of $x_{k}$ in the first step, we estimate all the elements of $\mathbf{x}_{k}$ in parallel as

$$
\begin{aligned}
& \mathbf{x}_{k}[n+1]= \\
& \left(\mathbf{R}_{k k} \mathbf{A}_{k}\right)^{-1}\left(\mathbf{y}_{k}-\sum_{j=1}^{k-1} \mathbf{R}_{k j} \mathbf{A}_{j} \mathbf{x}_{j}[n+1]-\sum_{j=k+1}^{K} \mathbf{R}_{k j} \mathbf{A}_{j} \mathbf{x}_{j}[n]\right)
\end{aligned}
$$

where $\mathbf{R}_{k j}$ and $\mathbf{A}_{k}$ are $M \times M$ block matrices. In the second step, the users' messages are obtained by using the same mapping as in (5).

\section{Partial Decorrelator}

Let the output of the full decorrelator [7] be

$$
\mathbf{z}=(\mathbf{R A})^{-1} \mathbf{y}=\mathbf{x}+(\mathbf{R A})^{-1} \mathbf{S}^{H} \mathbf{n}
$$

To obtain an estimate of each element $x_{k m}$ of $\mathbf{x}$, decorrelation is performed not only against all the other users' $M(K-1)$ signals, but also against the desired user's $M-1$ 
signals. Due to the total decorrelation strategy, this detector will be referred to as the full decorrelator.

It is interesting to note that in the full decorrelator, the desired user decorrelates against its other $M-1$ possible signals as well, even though we know that of the $M$ possible signals of the desired user, only one signal was sent. Hence, there is no need for the desired user to decorrelate against its other $M-1$ signals. In the proposed partial decorrelation scheme, decorrelation is performed only against the interferers' signals to obtain $\hat{x}_{k m}$. First, the vector $\overline{\mathbf{y}}_{k m}$ is constructed which only contains $y_{k m}$ and the $M(K-1)$ entries of $y$ belonging to the interferers. Similarly, the partial signature set $\overline{\mathbf{S}}_{k m}$ consists of the $k$ th user's signature corresponding to message $m$, and the $M(K-1)$ signatures corresponding to all the interferers' signals. The matrix $\overline{\mathbf{A}}_{k m}$ may be interpreted in a similar manner. The following steps implement the partial decorrelator.

$$
\begin{aligned}
\overline{\mathbf{z}}_{k m} & =\left(\overline{\mathbf{R}}_{k m} \overline{\mathbf{A}}_{k m}\right)^{-1} \overline{\mathbf{y}}_{k m} \\
& =\overline{\mathbf{x}}_{k m}+\left(\overline{\mathbf{R}}_{k m} \overline{\mathbf{A}}_{k m}\right)^{-1} \overline{\mathbf{S}}_{k m}^{H} \mathbf{n}
\end{aligned}
$$

The first entry of $\bar{z}_{k m}$ is equal to $\hat{x}_{k m}$. We perform the above partial decorrelative steps for all the messages of user $k$, and finally obtain $\hat{\mathbf{x}}_{k}=\left(\hat{x}_{k 1}, \ldots, \hat{x}_{k M}\right)^{\top}$. Then, the MM rule is applied to $\hat{\mathbf{x}}_{k}$ to obtain $\hat{m}_{k}$. The partial MMSE detector is obtained in a similar way. Note that if a particular user's signatures (associated with the $M$ messages) are mutually orthogonal, then the partial and full detectors are identical.

\section{Numerical Results and Discussions}

In all our simulations, we used the same random signature set that was used in [2]. The signatures are linearly independent and hence the inverse of the cross-correlation matrix $\mathbf{R}$ exists. User 1 is assumed to be the desired user, and in all the figures, $P_{s}$ represents the Probability of Symbol Error of user 1 . In all the simulations, the number of users $K=2$, the number of messages per user $M=4$, and the processing gain $N=20$.

Figure 1 plots $P_{s}$ versus the SNR of all the users (including the desired user) for the different detectors studied in this section. The local and global constrained detectors perform very close to the MMSE detector. This could be attributed to the resemblance of the analytical solutions (of constrained optimization problems) to the generalized MMSE solution. Figure 2 plots the $P_{s}$ of the desired user versus the SNR of the interferer in a near-far scenario.

Figure 3 compares the performance of the different SIC's proposed in this paper to the full decorrelative and MMSE detectors. In all the SICs, a decorrelative first stage was used followed by two more stages of processing of sufficient statistics. It is interesting to note that the MMSE detector does not converge to the decorrelator in the high interferer power region in contrast with multiuser systems that employ linear modulation, e.g., BPSK. This is a direct consequence of the fact that, in the near-far situation, the powers of the interferers are high compared to the powers associated with all possible messages of the desired user, and that the full detectors take the undesired $M-1$ messages of the desired user (with relatively low powers) as well all interferers signals (with high powers) into account in decoding the desired user's message. As a result, the MMSE detector does not zero-force the contributions of the $M-1$ undesired messages of the desired user as the decorrelator does. Hence the difference in the two in the near-far scenarios. However, this problem does not arise in the partial detectors, and the MMSE and decorrelator do converge in the near-far situations.

Figure 4 compares the performance of the partial and full detectors. The partial detectors consistently outperform the full detectors at all values of SNR, and at $P_{s}=10^{-3}$, the gap between them is around $1 \mathrm{~dB}$. Blind, adaptive implementations of the partial detectors are also currently under investigation.

\section{References}

[1] D. Bertsekas. Nonlinear Programming. Athena Scientific, 1995.

[2] A. Kapur, D. Das, and M. K. Varanasi. Noncoherent MMSE multiuser receivers for nonorthogonal multipulse modulation and blind adaptive algorithms. In CISS, Princeton University, March 2000.

[3] M. L. McCloud and L. L. Scharf. Asymptotic analysis of the MMSE multiuser detector for non-orthogonal multipulse modulation. IEEE Trans Comm. Submitted September 1999.

[4] L. B. Nelson and H. V. Poor. Iterative multiuser receivers for CDMA channels: An EM-based approach. IEEE Trans Comm, 44(12):1700-1710, December 1996.

[5] J. Proakis. Digital Communications. McGraw Hill, 3rd edition, 1995.

[6] M. K. Varanasi and B. Aazhang. Multistage detection in asynchronous CDMA communications. IEEE Trans Comm, 38(4):509-519, April 1990.

[7] M. K. Varanasi and A. Russ. Noncoherent decorrelative detection for nonorthogonal multipulse modulation over the multiuser gaussian channel. IEEE Trans Comm, 46(12):1675-1684, December 1998.

[8] S. Verdá. Multiuser Detection. Cambridge University Press, 1998.

[9] E. Visotsky and U. Madhow. Multiuser detection for CDMA systems with nonlinear modulation. Submitted.

[10] A. Yener, R. D. Yates, and S. Ulukus. CDMA multiuser detection: A nonlinear programming approach. IEEE Trans Comm. Submitted December 1999.

[11] A. Yener, R. D. Yates, and S. Ulukus. A nonlinear programming approach to CDMA multiuser detection. In In 33rd Asilomar Conference on Signals, Systems and Computers, October 1999. 


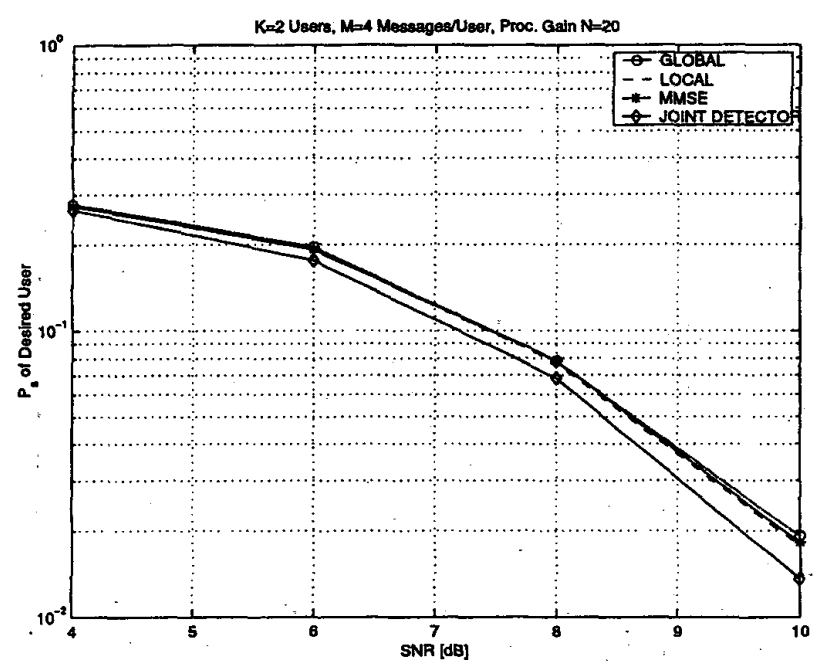

Figure 1. Comparison of various Noncoherent Detectors

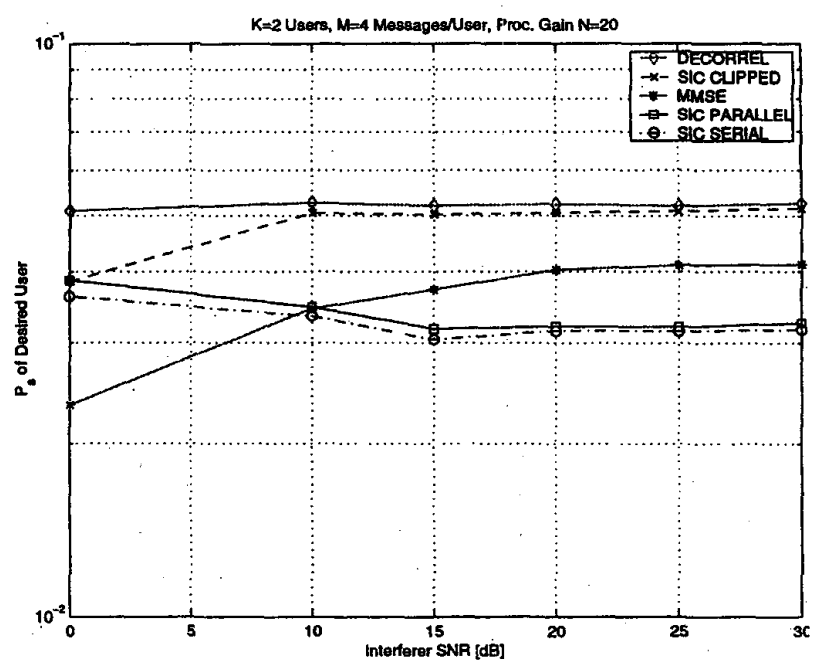

Figure 3. Comparison of SICs in Near-Far scenario. Desired user's $S N R=10 \mathrm{~dB}$.

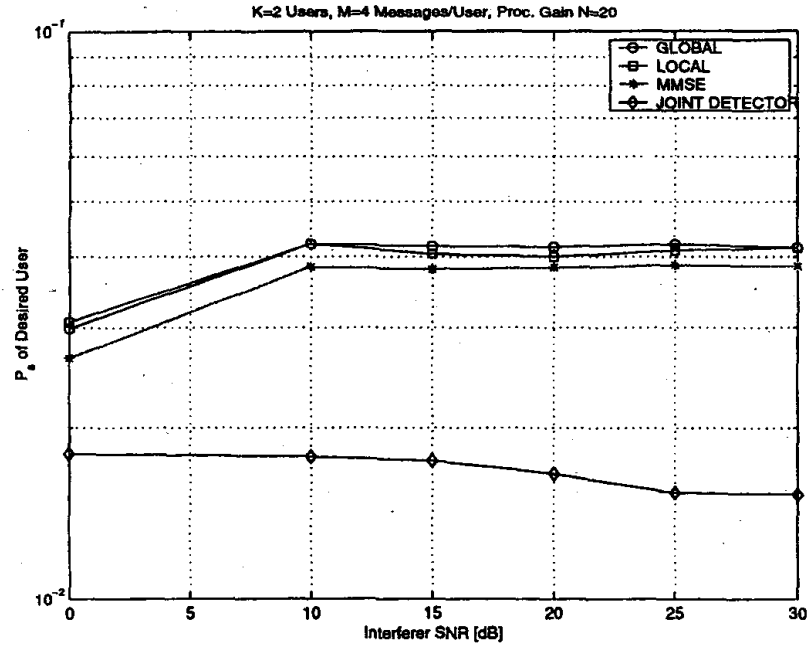

Figure 2. Comparison in the Near-Far scenario. Desired user's SNR $=10 \mathrm{~dB}$.

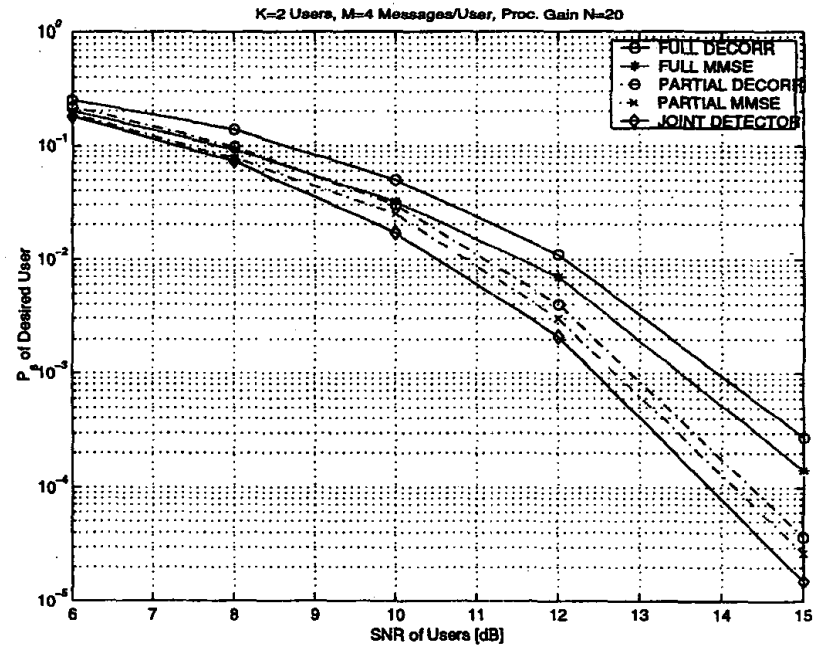

Figure 4. Comparison of the Partial and Full detectors. 